\title{
Design of an Agile Unmanned Combat Vehicle - A Product of the DARPA UGCV Program
}

\author{
Lindsey Thornhill', Alan Walls ${ }^{1}$, Ron Arkin ${ }^{2}$, Joe Beno ${ }^{3}$, Chuck Bergh ${ }^{4}$, Don Bresie ${ }^{3}$, Anthony \\ Giovannetti ${ }^{5}$, Benny Gothard ${ }^{1}$, Larry Matthies ${ }^{4}$, Porfirio Nogueiro ${ }^{5}$, Jim Scanlon ${ }^{1}$, Ron Scott ${ }^{1}$, \\ Miguel Simon ${ }^{1}$, Wilford Smith ${ }^{1}$, Ken Waldron ${ }^{6}$ \\ ' Science Applications International Corporation, 1410 Spring Hill Road, Suite 400, MS SH4-5, \\ McLean, VA 22102 \\ ${ }^{2}$ Georgia Institute of Technology, College of Computing, Atlanta, GA 30332 \\ ${ }^{3}$ University of Texas Center for Electromechanics, 10100 Burnett Road, Bldg 133, Austin, TX \\ 78758 \\ ${ }^{4}$ NASA Jet Propulsion Laboratory, California Institute of Technology, MS 125-209, Pasadena, \\ CA 91109 \\ ${ }^{5}$ United Defense, 1205 Coleman Avenue, Santa Clara, CA 95050 \\ ${ }^{6}$ Stanford University, Department of Mechanical Engineering, Design Division, Terman \\ Engineering Center 551, Stanford, CA 94305
}

\begin{abstract}
The unmanned ground combat vehicle (UGCV) design evolved by the SAIC team on the DARPA UGCV Program is summarized in this paper. This UGCV design provides exceptional performance against all of the program metrics and incorporates key attributes essential for high performance robotic combat vehicles. This performance includes protection against $7.62 \mathrm{~mm}$ threats, $\mathrm{C} 130$ and $\mathrm{CH} 47$ transportability, and the ability to accept several relevant weapons payloads, as well as advanced sensors and perception algorithms evolving from the PerceptOR program. The UGCV design incorporates a combination of technologies and design features, carefully selected through detailed trade studies, which provide optimum performance against mobility, payload, and endurance goals without sacrificing transportability, survivability, or life cycle cost. The design was optimized to maximize performance against all Category I metrics. In each case, the performance of this design was validated with detailed simulations, indicating that the vehicle exceeded the Category I metrics. Mobility metrics were analyzed using high fidelity VisualNastran vehicle models, which incorporate the suspension control algorithms and controller cycles times. DADS/Easy 5 3-D models and ADAMS simulations were also used to validate vehicle dynamics and control algorithms during obstacle negotiation.
\end{abstract}

Keywords: UGCV, mobility, unmanned, proprioception, robotics, active suspension

\section{INTRODUCTION}

The DARPA Unmanned Ground Combat Vehicle (UGCV) Program is aimed at producing both a weapons class and a reconnaissance class ground vehicle with unprecedented off-road mobility characteristics. The principal performance goals of the program, the so-called Category I Metrics, focus on three aspects of performance: endurance, obstacle negotiation, and payload fraction. The vehicles are expected to be capable of 14 day endurance with a range of $450 \mathrm{~km}$. They also are expected to be capable of negotiating $1 / 4$ meter obstacles at off-road speeds and negotiating 1 meter obstacles at reduced speed (or from halt). Finally, the vehicles are expected to achieve a payload fraction, the ratio of payload mass to gross vehicle weight, of at least 0.25 .

In addition to these Category I metrics, the program seeks to maximize the vehicles performance against a long list of less well-defined Category II metrics. These metrics include such performance characteristics as

- Maneuverability in confined spaces,

- Mobility in soft soils, 
- Stability on slippery side slopes,

- Mobility over rocky terrain,

- Swim capability,

- Resilience to bumps and crashes,

- Reliability,

- Ability to maintain sensor stability,

- Airlift and airdrop capability,

- Ability to engage in cooperative behaviors,

- Minimal signature, and

- Reasonable life-cycle costs.

Science Applications International Corporation (SAIC) assembled a team of experts in the various disciplines relevant to ground robotics to evolve an innovative design for the weapons class UGCV. This team was made up of key staff from Georgia Tech, Stanford University, the University of Texas Center for Electromechanics, the Jet Propulsion Laboratory, United Defense, and PreMag (makers of advanced, compact inverters), who provided expertise in a variety of areas as indicated in Figure 1. In addition to the roles indicated in this figure SAIC also provided program management, system engineering, and system integration expertise.

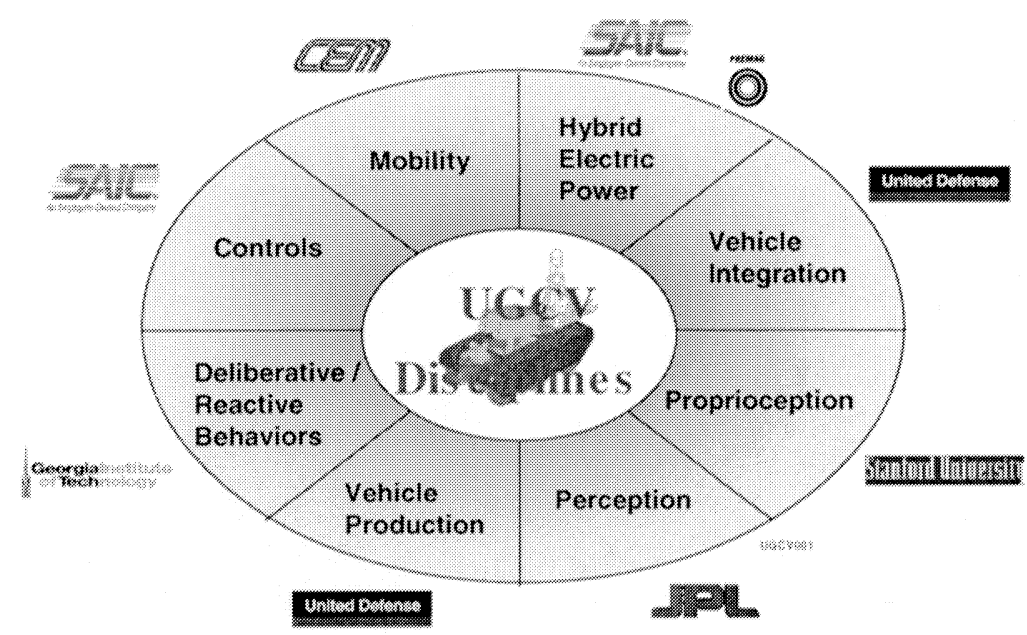

Figure 1. The SAIC UGCV Team included expertise in all disciplines relevant to ground robotics.

Throughout Phase 1A and Phase 1B of the UGCV Program this team completed the detailed design of our vehicle, and established all hardware and software interfaces. The team also completed the demonstration of all planned risk reduction experiments, to provide a high degree of confidence that the system would perform well upon roll out. These risk reduction experiments included successfully testing the engine/generator test stand at full power, demonstrating the wheel station and its controller operating and exceeding design goals, and demonstrating all control system hardware and software interfaces. Additionally, the team developed both high-fidelity and engineering-level vehicle models to aid in the design effort. The engineering models have been used to perform initial development of advanced behavioral algorithms (obstacle negotiation, cooperative vehicle behaviors, etc.). The high-fidelity models have been used for establishing wheel station control algorithms (anti-slip traction control, differential torque steering) and for testing and refinement of advanced behavioral algorithms using simulations with the vehicle controller in the loop.

The remainder of this paper is organized into three sections. In Section 2 a description of the UGCV system design is provided. The results of experiments aimed at reducing risk are reported in Section 3. Finally, a summary of the status of the design and testing effort is provided in Section 4. 


\section{SYSTEM DESIGN DESCRIPTION}

The 6x6 UGCV design evolved by the SAIC team, through extensive trade studies and through computational analyses and simulations, is illustrated in Figure 2 with the CKEM/OCSW weapon configuration. This design is $2.18 \mathrm{~m}$ in width and $3.8 \mathrm{~m}$ in length. In the nominal suspension position the distance from the ground to the top deck of the vehicle is $1.25 \mathrm{~m}$. In the full squat suspension position, the vehicle is approximately $1 \mathrm{~m}$ to the top deck. The vehicle is capable of full mobility at a gross vehicle weight of $6300 \mathrm{~kg}$, including a $1600 \mathrm{~kg}$ payload. The vehicle is capable of carrying payloads greater than $4500 \mathrm{~kg}$ with some reduction in mobility performance. Part of the gross vehicle weight results from the fact that the design provides complete, all-around protection from $7.62 \mathrm{~mm}$ weapons. This UGCV design is air drop compatible, $\mathrm{CH} 47$ and C130 transportable, capable of operation on $60 \%$ side slopes and swim capable.

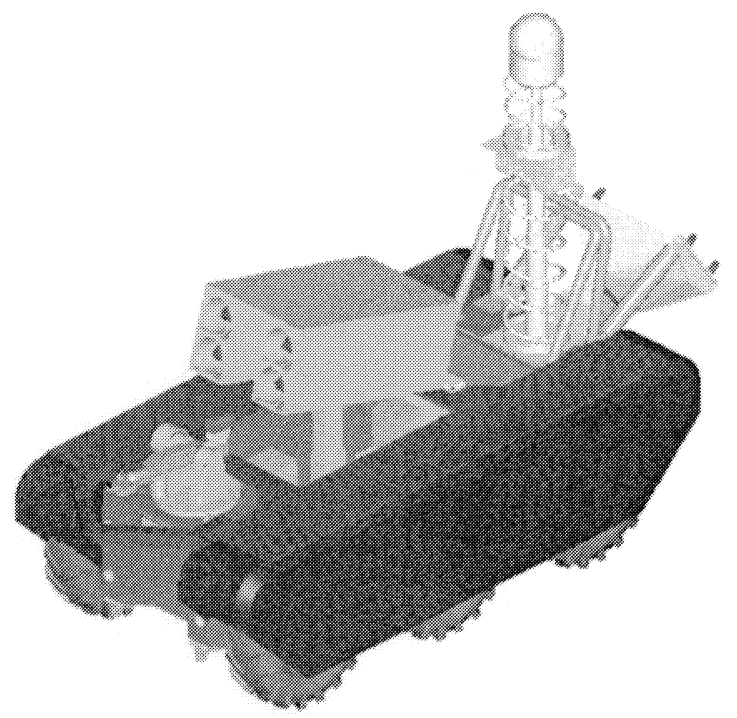

Figure 2. CKEM/OCSW variant of the UGCV design.

This design can sustain mobility at $96 \mathrm{kph}$ on improved roads, with a sprint capability up to $120 \mathrm{kph}$. On known, off-road terrain ("known" here meaning no known positive or negative obstacles of significance) the vehicle is capable of sustaining $72 \mathrm{kph}$. This speed represents a power limit against typical off-road rolling resistance. This 72 $\mathrm{kph}$ speed can also be sustained on a $2 \%$ slope. The vehicle is capable of sustaining $20 \mathrm{kph}$ in unknown off-road terrain, a speed that represents a perception limit, and it can sustain $7 \mathrm{kph}$ on $60 \%$ slopes. Finally, the vehicle carries enough fuel for 18 days operation based on typical mission profiles.

\subsection{Power and Thermal Management System Design}

The UGCV is powered by a fuel-efficient hybrid-electric power system, with the prime power provided by an 80 kW Volkswagen diesel engine driving a Unique Mobility Power Phase 100 generator. This compact, diesel-electric power-generation system provides sufficient power for continuous operation of all subsystems. All power users (including the propulsion system) are powered by electricity from a 270 volt DC bus. The energy storage system, which consists of a $15 \mathrm{~kW}$-hr Li-Ion battery pack, augments instantaneous system power so that power generation hardware can be sized to deliver only the average power. The energy storage system provides load leveling as well as power for silent watch and silent mobility. A power management system located within the vehicle control unit controls the flow between power generation, storage, and users for maximum fuel economy, stealth, or other criteria. A component view of the power system is shown in Figure 3. 


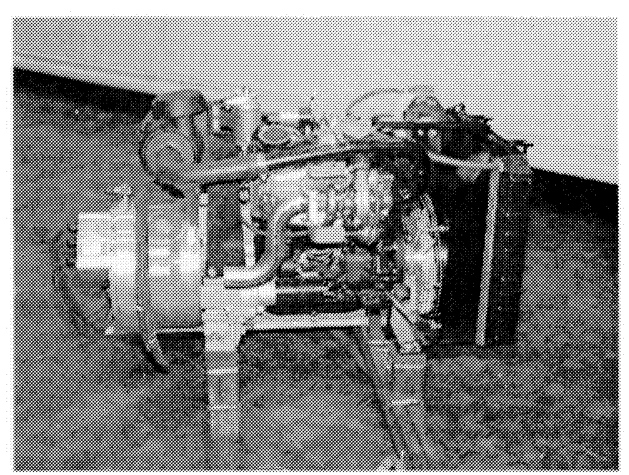

Figure 3. UGCV diesel-electric power generation system

The thermal management system is a conventional water/glycol system with a cooling loop for the engine, one for the generator, motors, and actuators, and a third for the charge air cooler. These three cooling loops are necessary to allow for different operating temperatures of the components. In this configuration the engine can operate at $105 \mathrm{C}$ while the generators and motors can operate at less than 60C. A schematic of the cooling circuit for the system is shown in Figure 4. The motor/generator circuit is designed to feed the three systems with a total cooling flow of between 14 and $56 \mathrm{gpm}$ of cooling fluid.

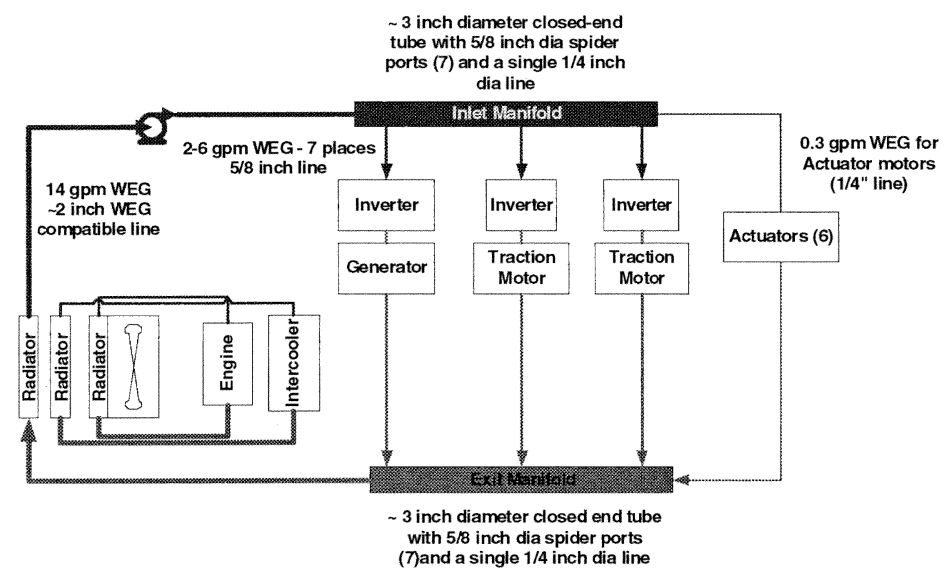

Figure 4. Schematic of cooling system design

\subsection{Mobility System Design}

The mobility system of the UGCV features a leading/trailing arm configuration with in-hub wheel motors and electromechanical, active suspension. These components make up 6 "wheel stations," which essentially include everything from the vehicle body to the ground, and a central control system that coordinates the operation of the 6 wheel stations. The wheel sation concept has been developed to minimize the volume dedicated to suspension and propulsion systems, to optimize platform stability for onboard sensors (especially sensors relevant to perception systems, the most taxing sensor related task), to maximize wheeled vehicle off-road and on-road performance, and to maximize commonality among the entire family of FCS manned and unmanned vehicles. Figure 5 illustrates the integration of technologies represented by the wheel station for the UGCV (as well as other FCS vehicles). 
Side View

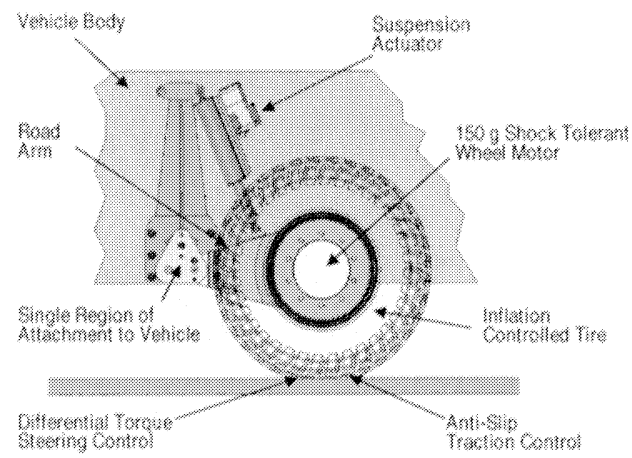

Rear View

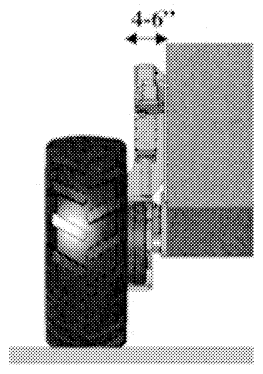

Figure 5. Wheel Station concept overview, with low volume, high performance design.

During UGCV Phase 1A and 1B, a complete design trade study was conducted to fully integrate suspension topology and linkage design with suspension actuator and wheel motor design. While the result appears to be a simple leading/trailing arm topology, important details such as passage of power and coolant lines through road arm bearings and transmitting suspension loads to the vehicle chassis strongly impacted wheel motor and actuator specifications and design. Trade studies indicated that other suspension topologies (such as four-bar, Modified Watt linkages) resulted in complicated vehicle integration issues resulting in larger volume and mass claims. The baseline design requires only 4 inches of space between the vehicle body and the tire and still allows up to 20 inches of suspension travel, a significant advantage in achieving high cross-country speeds.

The UTCEM active suspension system was developed and demonstrated at the prototype level by UTCEM and is being militarized and readied for production by Northrop Grumman-Litton. The system includes: (1) electromechanical actuators, with substantially higher bandwidth than typical hydraulic active suspension systems, (2) energy conserving features such as regenerative damping control and use of passive springs to supplement force actuators so that energy consumption is lower than hydraulic active suspension systems and, as demonstrated in Army testing, lower than typical losses associated with passive shock absorbers on off-road terrain, and (3) advanced control algorithms with performance exceeding previous active suspension systems. Army test results at Yuma Proving Grounds (YPG), comparing the UTCEM active suspension and conventional passive suspension in HMMWV vehicles, demonstrate at least a two-fold cross-country speed increase. With improved electronics a threefold increase could be achieved, coupled with a simultaneous 5-10 fold reduction in absorbed power, as shown in Figure 6. Additional tests demonstrated higher peak controllable speeds in slalom tests and CG height control during turns to reduce rollover probability. 

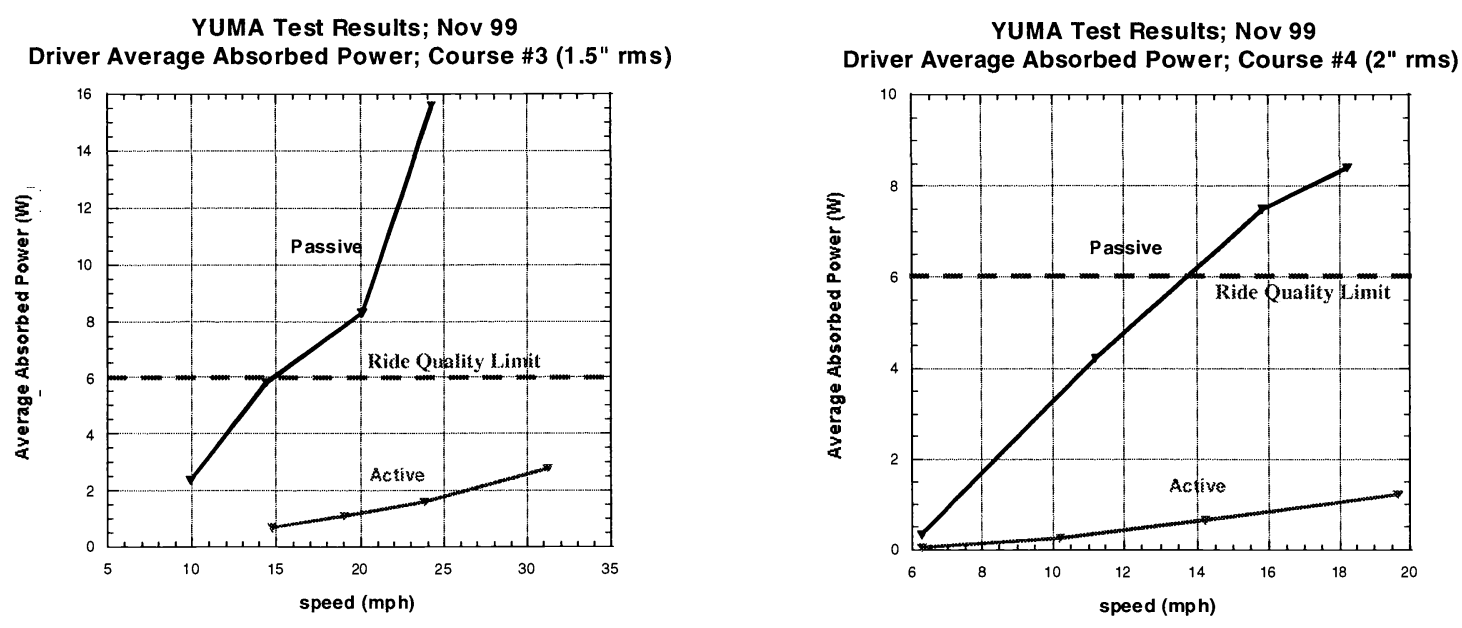

Figure 6. Army test results of UTCEM active suspension system.

The desire to maximize UGCV payload volume and payload mass fraction naturally leads to consideration of wheel motors. Wheel motors are particularly attractive for suspension systems with large wheel travel due to complications arising from power transfer to wheels. The increased cross-country speeds enabled by UTCEM's active suspension system, however, cause unsprung mass components (such as wheel motors) to be subjected to higher shock loading than the low-speed operation of passive systems. Suspension models indicate that log-strikes while traversing off-road terrain at the now higher speeds enabled can result in shock loading on unsprung mass components to exceed $100 \mathrm{~g}$ 's, leading to $150 \mathrm{~g}$ 's shock survivability as a suitable design goal for wheel motors. UTCEM developed optimized wheel motor designs that meet or exceed all UGCV performance needs, meet the 150 $\mathrm{g}$ requirement, and still equal or surpass existing wheel motor specifications for size and mass.

The UGCV design evolved by the SAIC team incorporates a principal benefit of independent wheel motors, which is the ability to use differential torque steering (DTS) rather than traditional Ackerman steering. This provides additional volume reductions for the suspension system (increasing payload volume), allows the complete elimination of the steering subsystem, and enables zero-radius turns. DTS requires good control of vehicle traction and will be degraded if tires transition in and out of sliding situations. Consequently, to be most beneficial DTS depends on an effective anti-slip traction control (ASTC), which involves sensing tire spin and taking appropriate actions for vehicle mobility and stability. Both DTS and ASTC are integral to the UGCV wheel station design. An additional feature of the UGCV wheel station design is active central tire inflation (ACTI). The ACTI controller employs sensors required by other wheel station subsystems, particularly active suspension and traction motors, as input to recognize when tire pressure should be increased or decreased for optimum trafficability and minimized rolling losses.

The UGCV wheel station control unit (WSCU) includes the hardware and software necessary to coordinate the operation of the 6 wheel stations so that vehicle propulsion and platform stability is optimized. For the roll-out vehicle, the WSCU was designed for a dSpace controller, minimizing risk and cost associated with transferring the WSCU control algorithms from simulation to actual UGCV vehicle hardware control systems. A significant accomplishment during Phase 1B involved configuring the entire WSCU to expand the current UTCEM suspension control system to include the full WSCU functionality while simultaneously reducing the controller cycle time by a factor of 10 .

\subsection{Hull Design}

The UGCV hull structure was designed to meet the weight allocation while providing a high level of ballistic protection and structural robustness at an affordable cost. The structure was designed to be fabricated from $19 \mathrm{~mm}$ thick titanium for the exterior plates. A front and rear interior bulkhead was designed using $5 \mathrm{~mm}$ thick titanium. This design meets all structural requirements and has the capability of defeating 7.62 AP all around. Other threats defeated by the $19 \mathrm{~mm}$ thick titanium plates include: 
- 0.30 caliber Ball M2 at muzzle velocity all around,

- 0.30 caliber AP M2 at muzzle velocity all around, and

- 0.50 caliber APM2 at 375 meters at 120 degree frontal arc.

Following an extensive analysis of options, titanium was again chosen for use on the bottom plate of the structure. The bottom plate of the hull structure was also sized at $19 \mathrm{~mm}$ thick. This design prevents plate rupture from a 0.6 $\mathrm{kg}$ explosive blast at nine inches from the ground. For comparison purposes, the Bradley Fighting Vehicle bottom plate is fabricated using 1.125 inches of 5083 aluminum and withstands blast loads and rupture from an M16A2 blast mine, which is $0.5 \mathrm{~kg}$ of explosive. Extensive modal and structural analyses of the hull structure under various static and dynamic loads were performed to validate its design. Typical results from these analyses are shown in Figure 7.

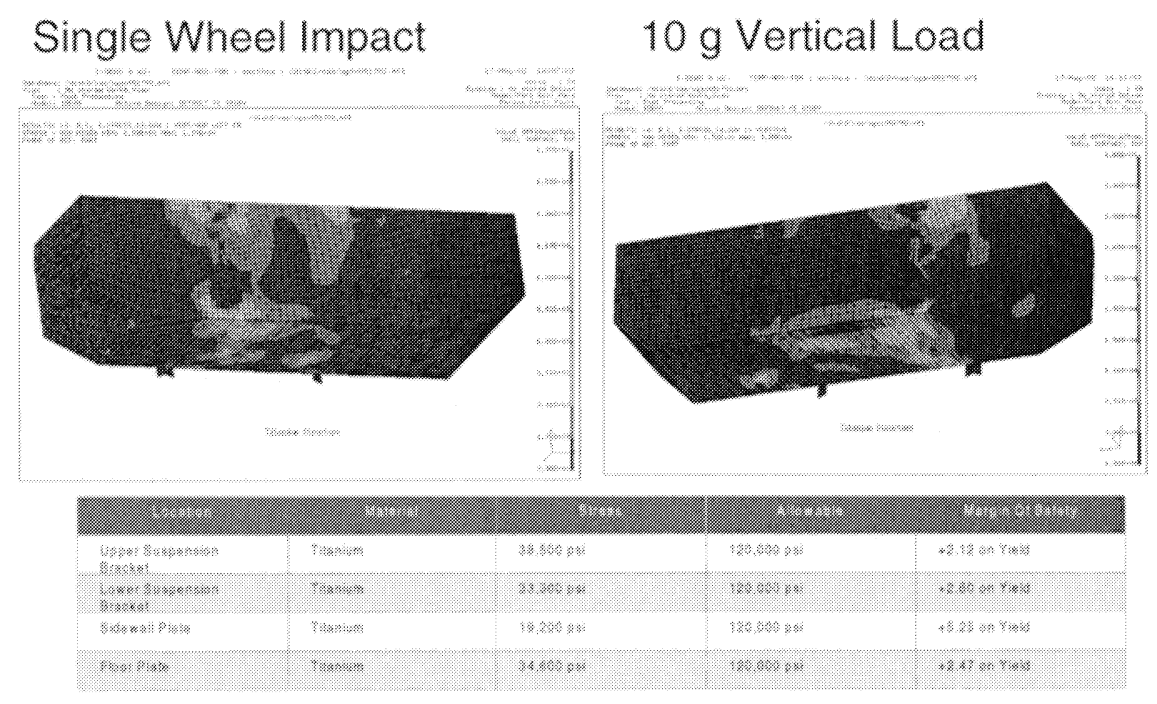

Figure 7. Typical results from from structural and modal analyses of the vehicle hull.

Another feature of the hull design is a bay incorporated into the front of the vehicle sufficient to accommodate two PackBot marsupial robots. The vehicle design included power and power coupling accommodations for recharging the PackBots and for deploying and receiving them with the vehicle in the full squat position. The marsupial bay is visible in the images in Figure 7. The hull design also features an active articulation joint for performing cooperative vehicle behaviors. The design for this joint was selected from several candidates as providing the greatest benefit-toweight/complexity ratio. By coupling two vehicles together DADS/ProE simulations indicate the coupled vehicles can negotiate positive steps of at least $1.75 \mathrm{~m}$ and trenches of $3.5 \mathrm{~m}$ width. The joint was designed to be folded against the vehicle when not in use. The joint, as well as a cooperative gap crossing, is illustrated in Figure 8 .
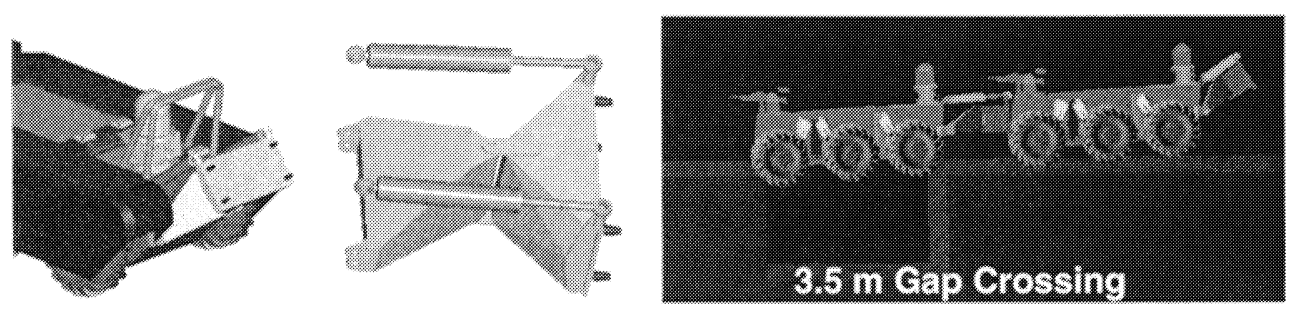

Figure 8. Active articulation joint and DADS/ProE simulation of trench crossing

The hull design also features multifunction fenders that provide performance enhancement in several of the Category II metrics. First, the fenders limit debris being thrown up to foul sensors and they protect surrounding personnel from being entangled in the wheel stations. Next, the fenders provide signature management by shielding thermal dissipation from the wheel stations and by minimizing radar cross sections. By incorporating inflatable 
swim bladders underneath the fenders, the vehicle is made swim capable. Finally, by actuating the fenders about a pivot at the rear of the vehicle, the rods supporting the fenders can be used to perform a self-righting maneuver. Frames from a DADS/ProE simulation of this maneuver are shown in Figure 9.

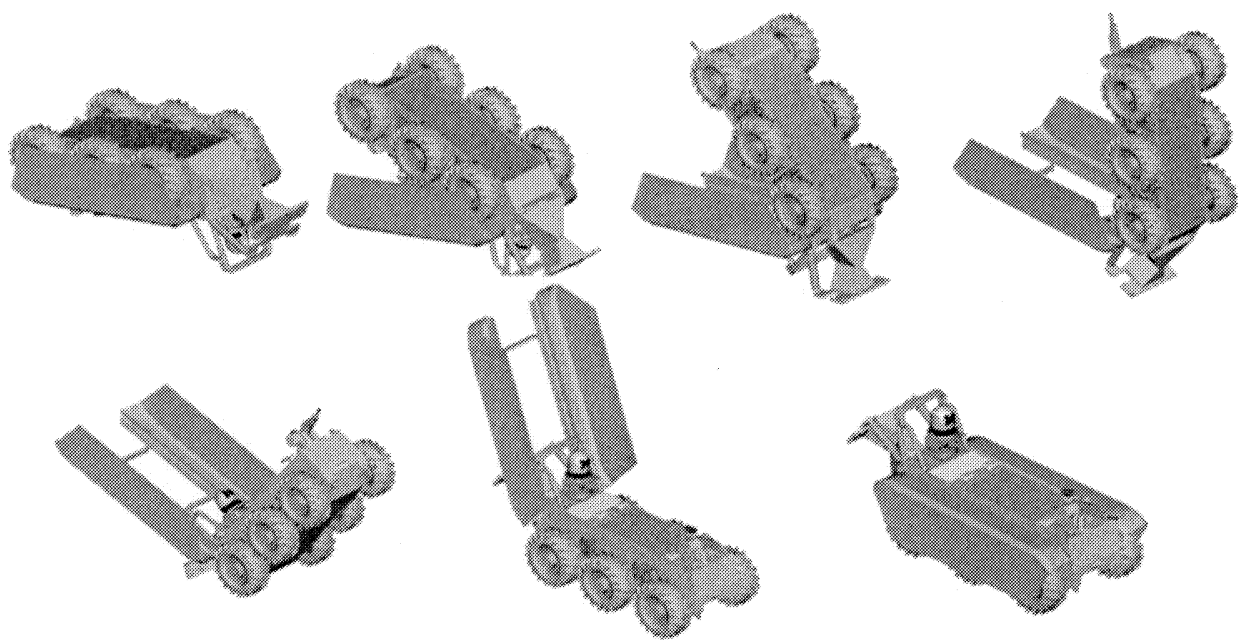

Figure 9. Self-righting maneuver is accomplished by actuating the mult-function fenders

\subsection{Control System}

The UGCV design features the Advanced Mobility Control System (AMCS) developed by the SAIC UGCV Team. The AMCS couples the deliberative/reactive, behavior-based control of the DARPA-funded MissionLab software, developed at Georgia Tech, with unprecedented levels of proprioception made possible by the integration of fully active and sensing wheel stations. The availability of extensive proprioceptive data is made possible by the suite of sensors integrated into the wheel stations. In addition to sensing, the active wheel stations enable the development of an unlimited number of behaviors in response to the proprioceptive sensing. This control system design leverages a wealth of research from previous and ongoing programs at both the hardware and software levels. It also enables the on-going development of new mobility behaviors using information gleaned from mobility tests. The control system hardware was built, and initial software implementation was installed and tested in the hardware. The SAIC UGCV team tested and validated interfaces throughout the full control system hierarchy. In addition, proprioceptive sequencing algorithms were validated with hardware-in-the-loop simulations driven by the control system.

The AMCS architecture, illustrated in Figure 9, is hierarchical, layered and modular, requiring a minimum of original software development. Each module of the architecture is self-contained, and as such, each module was debugged and tested independently to a great extent. This allows for concurrent development and testing of all the different control modules. The control architecture modularity also influences the ability to assign different degrees of reactive control and decision-making autonomy to different control layers. Each layer supervises and if necessary intervenes in the control actions and decision-making abilities of the layer below. 


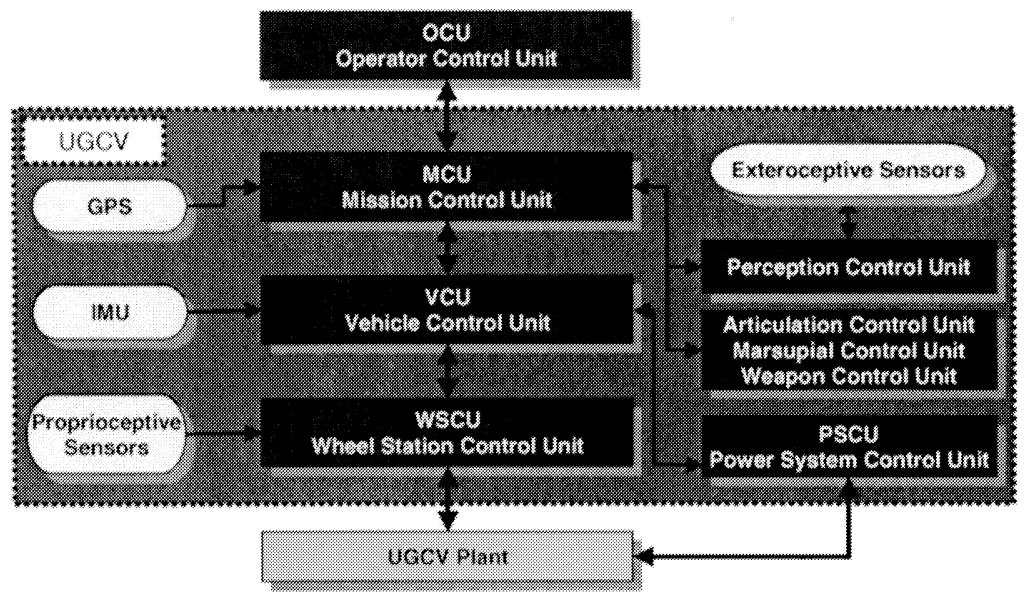

Figure 9. The Advanced Mobility Control System architecture

For example, mobility control exists at the bottom most layer of the architecture, and is implemented by the Wheel Station Control Unit (WSCU) module. This control module possesses a very high degree of reactive control along with sophisticated decision-making autonomy. WSCU autonomy is dictated by the mode of operation set by the Vehicle Control Unit (VCU) layer. In autonomous mode, the WSCU will react to changing conditions such as rough terrain and different traction conditions, and will maintain preset goals such as vehicle attitude and ride height. In this mode of operation, decision making authority includes the ability to decide which wheels are best suited to have maximum torque, which speed should the vehicle have given current traction conditions and such. In contrast, the WSCU can operate in slave mode, and in this mode, the WSCU surrenders autonomy to the VCU over such control elements as wheel torques, ride height, and suspension actuator force or position.

The VCU control layer oversees the performance of the WSCU. The VCU processes overall vehicle state data including data that originates from the Inertial Measurement Unit (IMU)- along with WSCU data, and generates proprioceptive features with this data. The VCU provides the infrastructure necessary to implement the lower level - and thus more reactive in nature- proprioceptive behaviors, which operate in real-time at a $100 \mathrm{~Hz}$-update rate. Proprioceptive behaviors, in turn, generate command inputs to control the WSCU torque, actuator and ride height settings when the WSCU operates in slave mode.

Reactive control is inherent to the proprioceptive control mechanism, and the degree of decsion making autonomy of the VCU control layer varies according to settings from the Mission Control Unit (MCU) which supervises the performance of the VCU. As such, the MCU manages the overall proprioceptive control mechanism along with the functionality of the Power System Control Unit (PSCU) by setting operating modes (i.e. attack, stealth, and cruising modes). Under each operating mode, several operations in the proprioceptive and PSCU algorithms become dominant and suppress, inhibit, enable or stimulate execution of a set of other subsystem functionalities. Whence in general, the VCU limits commands to the lower level WSCU so that stability is ensured, and it acts as a filter for normal operations and as a closed-loop control agent for large obstacle negotiation and other advanced behaviors. In turn, the PSCU manages power generator set-point and battery state to support mobility requirements. It also manages the thermal conditioning of all system components.

The MCU coordinates and commands control of the vehicle as a whole commanding heading, speed and vehicle mode. The MCU utilizes IMU and GPS data lumped into navigation features along with other exteroceptive data and operator commands to select appropriate operating modes and associated behaviors. Behaviors utilize the acquired knowledge to trigger conditions that control the behavior sequencing mechanism pertaining to each mode. In general, an active set of behaviors dominate the overall autonomous decision making mechanism of the control system, but even at this higher layer within the control hierarchy, reactive control is still very much present. For example, the operational mode setting and trigger actions that sequence a set of associated behaviors are themselves inherently reactive in nature since trigger conditions are themselves dependent upon dynamically changing conditions. The MCU runs the MissionLab executable code where the behavior sequencing actually takes place. 
The OCU serves as the human control interface to the UGCV. The OCU allows an operator to input and control an ongoing mission in a dynamic fashion. The OCU creates operations file based on an operator-selected path. The operations file includes waypoints and specific states for execution in MissionLab's CFG-edit. MissionLab's CDL generator translates the file into a mission, with start, wait-for-release, stop and a series of corresponding Go To states. During normal operations, control and status messages are passed between OCU and MCU at run-time using a message-based paradigm implemented in IPT. The OCU and MissionLab integration also provides the operator with means to download executable code to the vehicle, as well as visual and subsystem status information for the duration of a mission. In addition, the OCU provides base station differential GPS information to the real-time kinematics unit mounted in the actual vehicle.

The AMCS control architecture minimizes the requirements for original software and hardware development by building upon existing programs. The OCU and MCU features DARPA-funded MissionLab software running on the MCU, which coordinates command and control of the vehicle. Proprioceptive data fusion and subsequent command generation resides in the VCU. The OCU builds upon SAIC's IR\&D efforts and upon results from programs such as DARPA's PercetOR. In turn, MissionLab leverages efforts expended in DARPA FCS-C and MARS programs. The WSCU is an extension and enhancement of the active EM suspension controls developed on DARPA and Army programs, and the PSCU is derived from the control system developed for the DARPA Combat Hybrid Power System Program. Finally, the PCU is being harvested from several DARPA and Army programs, including TMR, DEMO II and DEMO III.

\subsection{Perception System}

The goals of the perception system in the UGCV program were to provide the MCU feed-forward knowledge of the upcoming terrain, to enrich the knowledge available to the user for tele-operating the vehicle, and to provide a foundation for the future integration of developing perception systems. Our perception system design met these goals by using existing sensors and algorithms. This approach was taken to lower the technical risk and cost by reusing successfully demonstrated algorithms and utilizing commercial off-the-shelf components. The proposed system consists of a passive stereo imaging system and an active one-axis scanning laser rangefinder mounted to the front of the vehicle. A RSTA package can be mounted to a deployable mast. Figure 10 shows the location of the sensors on the vehicle.

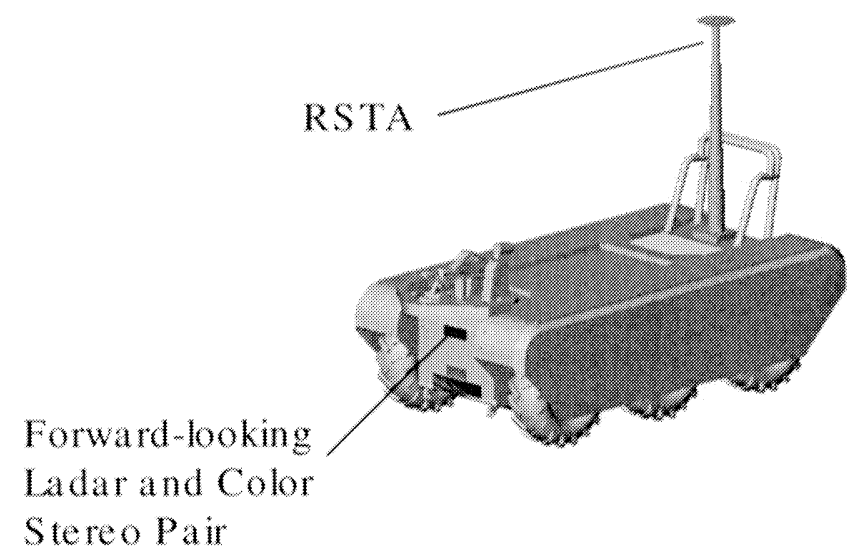

Figure 10. Phantom vehicle with perception elements called out

In programs such as PerceptOR, Tactical Mobile Robots (TMR), and Demo III, we used a real-time, passive stereo imaging system to perceive terrain geometry in terms of range and elevation, to generate a list of obstacles and location, and to infer the basic composition of the terrain. Point Grey Research Dragonfly cameras with 640x480 pixels with $90^{\circ} \mathrm{FOV}$ lenses were planned for stereo range acquisition under day-time, medium to high visibility conditions. These cameras were developed in the TMR program and have SOA features such as a digital interface (IEEE-1394) and automatic camera-to-camera synchronization. A pair of these cameras will be housed in an environmental enclosure in the forward section of the vehicle. 
A single-axis ladar, SICK LMS200 with $180^{\circ} \mathrm{FOV}$, was planned for generateing a $2 \mathrm{D}$ terrain profile along the direction of travel. The unit was planned to be mounted vertically on the vehicle centerline and operates at $40 \mathrm{~Hz}$ with $0.5^{\circ}$ resolution and with $300 \mathrm{~m}$ range on natural targets. This approach was chosen to provide high-resolution terrain data at high frequencies to the suspension controller while also providing redundancy to the stereo ranging system and extending operation into lower visibility conditions. With data being taken along the vehicle centerline, it is assumed that each wheel track will see similar terrain. While a two-axis ladar has the ability to generate range and elevation maps similar to those produced by stereovision, the cost was prohibitive for this program. SICK scanners have been used in many different perception applications in TMR and PerceptOR and have been proven to be durable, reliable sensors.

Provisions were included in the design for the mounting and interfacing of a Wescam 14PS RSTA package. A mast is used to meet the two opposing goals of signature management and sensor placement for maximum field of view. FCS recommends that a RSTA package be placed at least $3 \mathrm{~m}$ above level ground in order to achieve maximum situational awareness. When the target sensor is not needed, or when the vehicle silhouette needs to be reduced, the mast could be retracted to stow the sensor in a safe, but functional, location. A modified Will-Burt Sabre mast with 5 sections was selected to provide $3 \mathrm{~m}$ of lift for payloads up to $200 \mathrm{~kg}$. For long-term durability, the mast will be housed within the vehicle hull.

\section{RESULTS OF RISK REDUCTION EXPERIMENTS}

In Phase 1B of the UGCV Program a series of risk reduction experiments were conducted to demonstrate various hardware and software components of the design. The first of these experiments demonstrated the operation of the power system. In this experiment the engine and generator were integrated and powered to run a dummy load. This experiment also assessed the performance of principal elements of the thermal management system. The power system was fully constructed on a skid that could be dropped into the vehicle. Typical data from operating the Power System Test Stand is shown in Figure 11 where we show the power capability of the system up to $\sim 40 \mathrm{~kW}$.
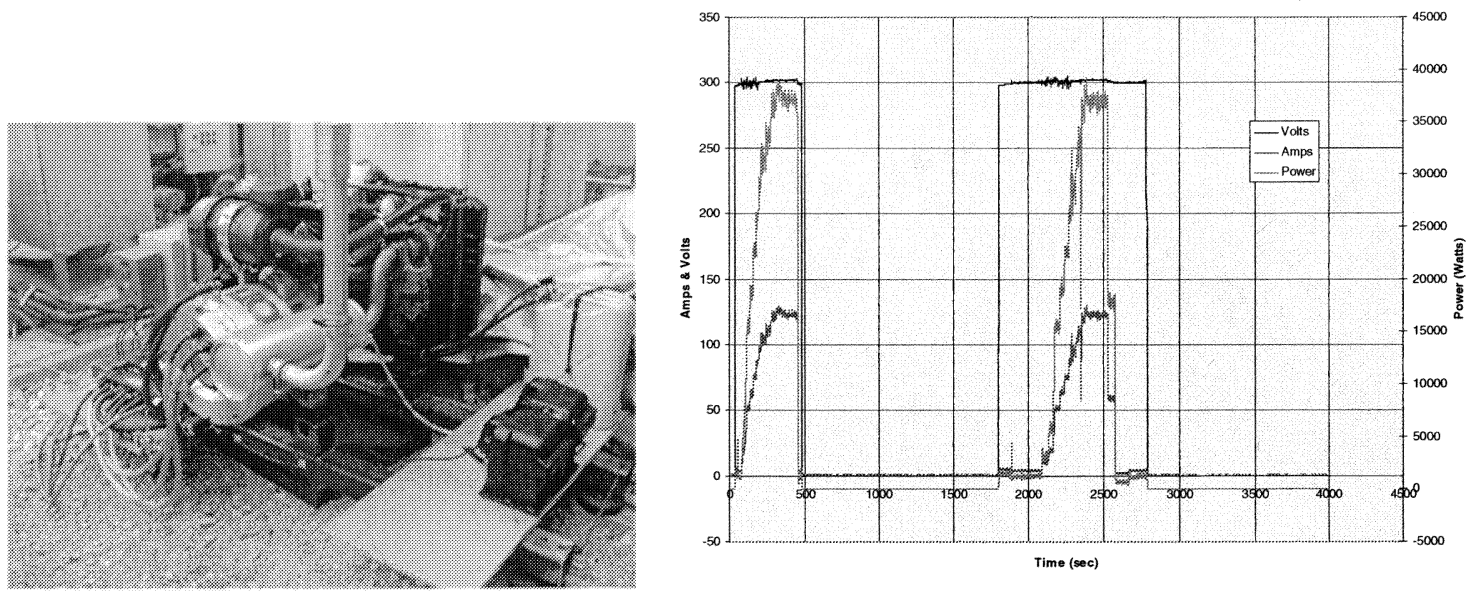

Figure 11. The power system test stand validated power generation performance and thermal management

To validate the design and operation of the wheel stations a wheel station test stand was constructed. The full-scale demonstration, shown in Figure 12, included one complete set of wheel station hardware and one WSCU. Full wheel station functionality was demonstrated on the test stand. Specific goals and accomplishments from this activity are listed in Table 1 . Although the WSCU only controlled one wheel station in this test, all computations required for the $6 \times 6 \mathrm{UGCV}$ were accomplished, fully demonstrating the viability of the hardware and software design. 

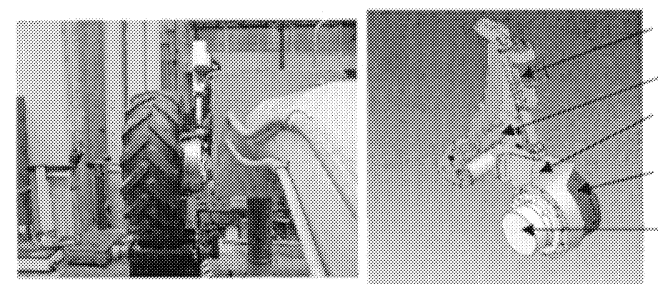

Fus Swotivg

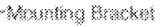

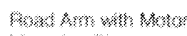

Wwank

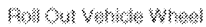

What

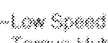

Trexus the
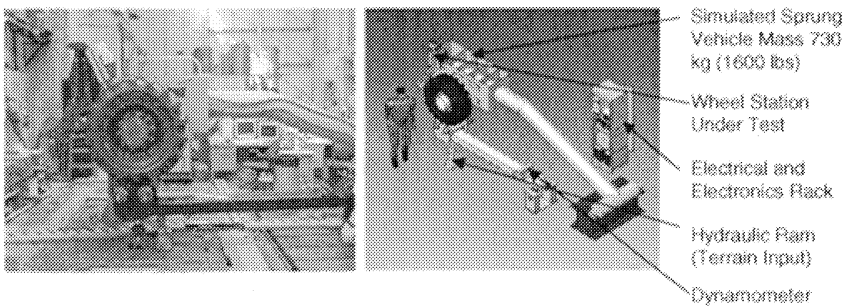

Figure 12. The wheel station test stand demonstrated full functionality of a single wheel station

Table 1. Goals and accomplishments of wheel station test stand

\begin{tabular}{|c|c|c|}
\hline Subsystem & Major Risk Mitigation Activity for PH IB & Accomplishments \\
\hline Suspension Actuator & $\begin{array}{l}\text { Needed } 50 \% \text { increase in peak force plus adjustable } \\
\text { ride height (net } 200 \% \text { increase in force). }\end{array}$ & $\begin{array}{l}\text { Achieved net } 250 \% \text { increase in design; tested to } \\
170 \% \text { with further testing planned after debugging } \\
\text { ride height control. }\end{array}$ \\
\hline Suspension Actuator & $\begin{array}{l}\text { Desired to be compatible with Wheel-to-body } \\
\text { distance of } 4 \text { inches. }\end{array}$ & Achieved 4 inch goal. \\
\hline $\begin{array}{l}\text { Wheel Station Control } \\
\text { Unit }\end{array}$ & $\begin{array}{l}\text { Added } 6 \times 6 \text { suspension control, plus ASTC, DTS, } \\
\text { Ride height control, and slave modes in } 1 \text { controller } \\
\text { with less than } 2 \mathrm{~ms} \text { cycle time. }\end{array}$ & $\begin{array}{l}\text { Demonstrated WSCU cycle time } 120 \text { micro sec } \\
\text { with full WSCU adgonithms. }\end{array}$ \\
\hline WSCU & Interface with VCU. & Achieved initial demonstration \\
\hline \begin{tabular}{|l|} 
Suspension Topology - \\
Roadarm design
\end{tabular} & $\begin{array}{l}\text { Addressing acceptable whel to body distance, } \\
\text { pass throughs elc. planned for Phase II. }\end{array}$ & $\begin{array}{l}\text { Exceeded plans with fully functional first generation } \\
\text { on test rig. }\end{array}$ \\
\hline ASTC and DTS & $\begin{array}{l}\text { Planned for CHPS DTS program, addressed } \\
\text { primarily in simulation. }\end{array}$ & $\begin{array}{l}\text { Simulation completed. initial ASTC bas ed on INS } \\
\text { developed. }\end{array}$ \\
\hline \begin{tabular}{|l}
$\begin{array}{l}\text { Integrated Wheel } \\
\text { Station }\end{array}$ \\
\end{tabular} & $\begin{array}{l}\text { Physical integrity, integration design, tool for } \\
\text { WSCU development }\end{array}$ & Demonstrated \\
\hline ACTI & No plans for UGCV; upgrade opportunity only. & $\begin{array}{l}\text { Under separate funding developed tire deflection } \\
\text { sensor concept. Won FCS BIA funding to } \\
\text { advance } \Lambda \text { CTl technology. }\end{array}$ \\
\hline Wheel Station W eight & $\begin{array}{l}\text { Verily Wheel Station objective system weight of } \\
650 \mathrm{lbs} \text {. }\end{array}$ & $\begin{array}{l}\text { Achieved Wheel Station Weight of } x x x \times \text { lbs. with } \\
\text { pah to } 630 \text { lbs specified (objective wheel motors, } \\
\text { materials subs titution, etc.). }\end{array}$ \\
\hline
\end{tabular}

Finally, tests were performed to validate and demonstrate processor interfaces, control algorithms, and software development procedures for the AMCS. The tests involved both desktop PCs and the deliverable control components in a cPCI chassis. The test sequence involved the following events:

- Validation of OCU interface to MissionLab-Create mission description on OCU and compile to generate MissionLab executable. Run executable on same OCU hardware and execute mission using MissionLab internal vehicle model. Compare results with original OCU mission description.

- Validation of MissionLab execution in MCU hardware-Create mission description on OCU and compile to generate MissionLab executable. Install executable on MCU and execute mission using MissionLab internal vehicle model. Compare results with original OCU mission description.

- Processor-in-the-Loop test of VCU control logic - Generate executable code for VCU control logic with Real Time Workshop. Install code with QNX wrapper on deliverable VCU processor board within CompactPCI chassis. Interface VCU to desktop PC via Ethernet. Engage vehicle in normal mode command sequence using the vehicle model. Compare output with two preceding events. Acceptable comparisons confirmed validity of executable code and wrapper. Also confirmed that executable code completes each cycle in the required time interval since the data transfer to VCU and start of cycle were triggered by the simulation clock. This test is illustrated in Figure 13.

- Validation of MCU interface to VCU-Starting with hardware setup for previous event, remove Simulink description of normal mode command sequence. Develop MissionLab representation of normal mode command sequence. Install MissionLab on MCU with command sequence. Interface MCU to VCU over PCI bus. Engage MCU, VCU, and desktop PC in normal mode sequence. Compare results with three preceding events. Acceptable comparison confirmed MCU interface to VCU 
- Validation of GPS message synthesis and integration with Smith Industries Inertial Measurement Unit (IMU) Initial validation of IMU interface with RT-STAR GPS system was proven on PerceptOR. This exercise validated the GPS message synthesis for IMU integrated navigation solution. The same navigation solution is suitable for UGCV implementation and deployment of both rollout and objective vehicle.

- Validation of VCU interface to WSCU via RS232-Initial validation of VCU interface software using serial port installed in the CompactPCI chassis with the VCU processor board. Ship chassis to CEM and demonstrate interface to dSpace hardware operating in slave mode.

- Validation of VCU interface to WSCU via CAN BUS-Initial validation of VCU interface software using CAN BUS installed in the CompactPCI chassis with the VCU processor board.

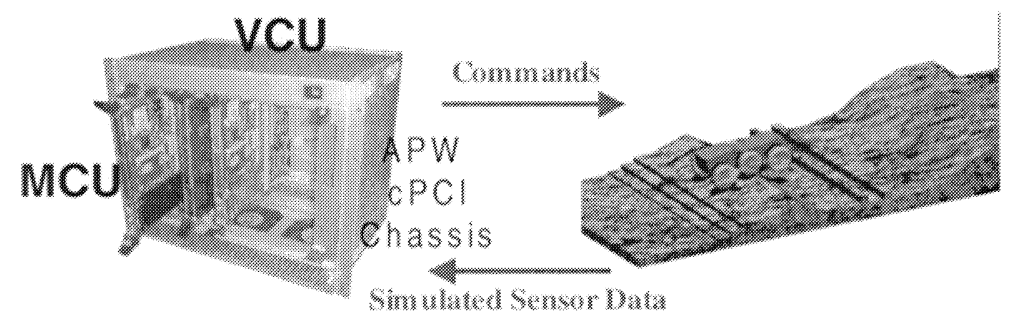

Figure 13. Vehicle simulations were used to demonstrate interfaces and algorithms in the VCU for $1 \mathrm{~m}$ obstacle crossing

The positive results from each of the tests described above indicated that each processor could perform a representative task and communicate with its neighbors as required. Since interfaces are the most troublesome issue in hardware/software integration, this exercise greatly reduced the design risk. The exercise also generated another positive result, the tools and the processes necessary to test and validate additions and changes to the baseline control implementation.

\section{SUMMARY}

The SAIC Team evolved a design for the UGCV that met or exceeded all of the Category I metrics. The design also enabled exceptional performance against the Category II metrics of the program. Risk reduction tests during Phase 1B validated the power generation and associated thermal management system. These tests also validated the hardware, software and interfaces in the wheel station design. Also in Phase 1B, each element of the AMCS was demonstrated along with the interfaces between elements. The synergistic combination of behavior-based controls with fully active and sensing wheel stations through the AMCS in this design represents a significant advance in offroad mobility systems. Sensors in the wheel stations provide a wealth of information about the state of mobility, and fully active wheel stations enable many degrees of freedom for taking action in response to state information. Not only does this design represent robust mobility upon roll out, it can continue to be refined indefinitely with additional behaviors and emergent behaviors as experience is gained with the vehicle in the field.

\section{ACKNOWLEDGEMENTS}

The authors gratefully acknowledge the funding for this effort provided by the DARPA UGCV Program and the guidance provided by the program manager Dr. Scott Fish. Additional funding for this work was provided by SAIC IR\&D and by United Defense IR\&D. 